\title{
Arachnoid cyst at upper lumbar region - A case report
}

\author{
*MMA Farazi', I Khalil'2, M Rashid ${ }^{3}$, SMI Kabir ${ }^{4}$, FB Moonmoon ${ }^{5}$
}

\begin{abstract}
A young male patient of 22 years was presented with the complaints of low back pain, hypoesthesia and inability to walk. MRI of lumbosacral spine reveals intradural mass lesion extending from L2-L3 with no enhancement of gadolinium. Hence an extensive laminectomy and total removal of the mass was done. Peroperative impression confirms arachnoid cyst. After operation there was dramatic improvement of signs and symptoms; patient was able to walk without support during discharge from the hospital after one week.
\end{abstract}

Keywords: Arachnoid cyst, Intradural, Laminectomy.

\section{Introduction}

Intradural extramedullary tumors constitute two-thirds of all spinal neoplasms. Schwannoma and meningiomas make up approximately $90 \%$ of the total number of neoplasms and occur in equal numbers. Spinal arachnoid cyst is a rare intradural tumor. Only $10 \%$ constitute ependymoma, dermoid, epidermoid, angioma, lipoma, metastatic carcinoma, arachnoid cyst, chordoma, lymphoma, melanoma, myxoma and sarcoma. Primary spinal tumors occur with an annual incidence of 2/100000 population. Male and female populations are equally affected and there is no age limitation. ${ }^{1}$ However, with the increasing availability of new radiological tools such as MRI (Magnetic resonance imaging), spinal cord tumors are discovered more frequently. ${ }^{2}$ Approximately $20 \%$ of all central nervous system tumors lie within the spinal canal. Estimates of location suggests that $25 \%$ are extradural, $50 \%$ are intradural extramedullary, and $25 \%$ are intramedullary. ${ }^{3}$ Clinical presentation includes radicular pain, definite sensory level, weakness of the limbs often associated with hyporeflexia and flaccidity and occasional bowel \& bladder involvement. Clinical presentation is useful in determining the exact location and level of the spinal lesion. ${ }^{4} \mathrm{MRI}$ (Magnetic resonance imaging) has become the primary diagnostic modality in the assessment of spinal lesion. ${ }^{5-7}$ Sometimes plain x-ray and CT myelogram may be helpful.

1. Dr. Md. Mohsin Ali Farazi, Associate Professor, Department of Neurosurgery, Shaheed Sheikh Abu Naser Specialized Hospital, Khulna. Email: mohsinfarazi@yahoo.com.

2. Dr. Ibrahim Khalil, Junior Consultant, Department of Neurosurgery, Shaheed Sheikh Abu Naser Specialized Hospital, Khulna.

3. Dr. Mamunur Rashid, Associate professor, Department of Anesthesiology, Gazi Medical College Hospital, Khulna.

4. Dr. S.M. Ikbal Kabir, Assistant professor, Department of Anesthesiology, Gazi Medical College Hospital, Khulna.

5. Dr. Farhana Boby Moonmoon, Medical officer, Department of Surgery, Gazi Medical College Hospital, Khulna. 


\section{Case history}

A 22 years male presented with low back pain, sudden weakness of both lower limbs making him unable to walk for 1 month. Muscle power was grade-2 in all groups of both lower limbs and grade- 5 in both upper limbs, DTR of ankle and knee were decreased with bilateral planter flexion. The sensory deficit was at L2 level bilaterally. There was no history of trauma, urinary and fecal incontinence but fever for few days. His x-ray chest was normal, ESR was $10 \mathrm{~mm}$ in first hour, $\mathrm{Hb} \%$ was $11.5 \mathrm{gm} / \mathrm{dl}$, serum creatinine level and ECG were normal. MRI of lumbosacral region showed intradural lesion with no enhancement of gadolinium extending from L2 to L3 level (Figure 02 and 03).
Wide laminectomy of L2 and L3 was done. After dural incision there was a cystic lesion within the arachnoid membrane. The lesion caused compression of the nerve roots from right to left. The lesion was ruptured during attempt of separation. The cystic fluid was clear and colorless like CSF. Watertight closer of dura was done and the wound was closed in layers keeping a drain in situ. Histopathological examination was not done. After operation signs and symptoms were gradually improved, physiotherapy was started and the patient was discharged on eighth post-operative day. During discharge the patient was symptom free and could walk without support. He was advised to take physiotherapy and light exercise regularly and also was followed up after one month.

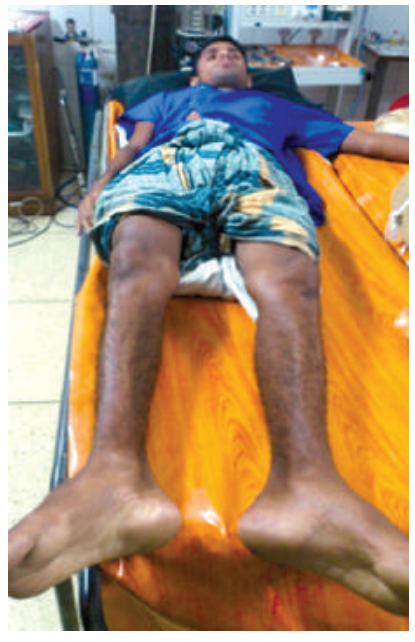

Figure 01: Muscle wasting in the lower limbs

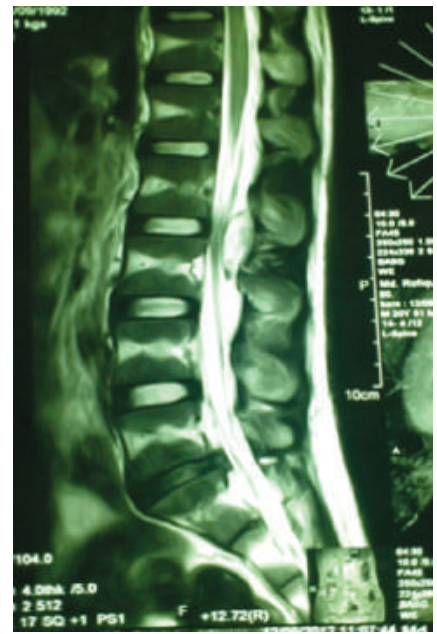

Figure 02: MRI T2

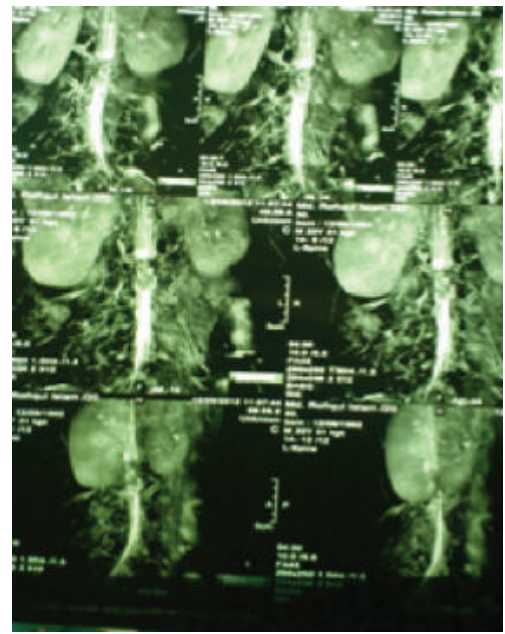

Figure 03: MR Myelogram

\section{Discussion}

Intradural arachnoid cysts are rare spinal tumor in lumbar region, particularly in upper level. It constitutes only $1-2 \%$ of all spinal tumors. Schwannoma and meningiomas constitute $90 \%$ and occur in equal numbers. 30-60 years of age groups are more prone to develop these conditions. Our patient was 22 years old. Pain and weakness are most readily identified objective symptoms and signs.
Radicular pain is often worse at night and may completely disappear at daytime. Weakness is always associated with hyporeflxia and flaccidity of the affected limbs (Figure 01).

MRI has become the primary diagnostic modality in the assessment of intradural lesions. Arachnoid cysts are hypointense on T1 weighted images but hyperintense on T2 weighted images. These lesions were non-enhanced with gadolinium. ${ }^{8}$ Histopathological examination was not done. 
Surgery is the treatment of choice. Short term outcome in the surgical tratment of intradural neoplasm is generally excellent with very gratifying improvement of neurologic function. ${ }^{9}$ Most patients will return to normal or near normal status. Physical therapy and occupational therapy are considered to be standard post operative treatment to optimize the neurologic recovery period. The risk of recurrence is estimated to be less than $10 \%$ in gross total resection of arachnoid cyst. ${ }^{10} \mathrm{~A}$ follow up $\mathrm{MRI}$ is recommended at 6 months and then 1 year after surgery. If there is no obvious recurrence, a follow up study is scheduled after 2 years. If the subsequent study does not demonstrate tumor recurrence, another follow up study at 5 years is recommended.

Complications related to surgical intervention for intradural tumors include hemorrhage, infection, CSF leakage and new significant neurodeficits. The post-operative sequele was uneventful in our case.

\section{Conclusion}

The surgical management of spinal arachnoid cyst has become simplified in the past 12 years. The excellent surgical outcome obtained today is primarily related to the early diagnosis achieved with new radiological diagnostic studies (MRI) and improved surgical techniques. In addition, intraoperative electrophysiologic monitoring has been exceedingly valuable in predicting, detecting and preventing neurologic injury. In summary, neurosurgeons today can anticipate a very satisfying surgical result in the treatment of arachnoid cysts.

\section{References}

1. Levy W, Latchaw J, Hahn J, et al. Spinal neurofibroma: a report of 66 cases and a comparison with meningiomas. Neurosurgery 1986; $18: 331-4$.
2. Namer IJ, Pamir MN, Benli K, Saglam S, Erbengi A. Spinal meningiomas. Neurochirurgia 1987; 30: 11-15.

3. McCormick P, Post K, Stein B. Intradural extramedullary tumors in adults. Neurosurg Clin North Am 1990; 1: 592-608.

4. Abernathey CD, Onofrio BM, Schneithauer BW, Pairolero PC, Shives TC. Surgical management of giant sacral schwannomas. J Neurosurg 1986, 65:286-95.

5. Dodge HW Jr, Svien H, Camp J, Craig W. Tumors of the spinal cord without neurologic manifestation producing low back pain sciatic pain. Proc Staff Meet Mayo Clin 1951; 26: 88.

6. Horrax G, Proppen JL, Wu WQ, Weadon $P R$. Meningiomas and neurofibromas of the spinal cord: certain clinical features and end results.Surg Clin North Am 1949; 29:659-65.

7. Blews D, Wang $\mathrm{H}$, Ashok $\mathrm{J}$, et al. Intradural spinal metastases in pediatric patients with primary intracranial neoplasms: Gd-DTPA-enhanced MR vs CT myelography. J Comput Assist Tomogr. 1990; 14: 730-5.

8. Sze G. Neoplastic disease of the spinal cord. In: Atlas SW (ed.),Magnetic Resonance Imaging of the Brain and Spine. Philadelphia: Lippincott Williams \& Wilkins;2002: 1715-67.

9. Ciapetta P, Domenicucci M, Raco M. Spinal meningiomas: prognosis and recovery factors in 22 cases with severe motor deficits. Acta neurol Scand 1988; 77: 27-30.

10. Klekamp J, Samii M. Surgical results for spinal meningi 